\title{
Repair of medial meniscus root tear could prevent progression to arthritis and subsequent knee arthroplasty
}

Christopher D Bernard, Nicholas I Kennedy, Adam J Tagliero, Christopher L Camp, Daniel B.F Saris, Bruce A Levy, Michael J Stuart, Aaron J Krych

\section{Video Abstract}

Keywords: medial meniscus, root tear, arthritis, knee arthroplasty, partial meniscectomy, axial loads, knee, biomechanical function, surgery, transtibial pull-through technique, Tegner scores, Kellgren-Lawrence grade, repair

Posted Date: February 14th, 2020

DOI: https://doi.org/10.21203/rs.2.23781/v1

License: (c) (i) This work is licensed under a Creative Commons Attribution 4.0 International License. Read Full License 


\section{Abstract}

The root of the medial meniscus is a vital component that provides stability and cushions axial loads to protect the knee. For that reason, meniscal root tears can be especially debilitating; studies have shown that tearing the posterior root of the medial meniscus is functionally and biomechanically comparable to a complete meniscectomy. Historically, such tears were treated without surgery or with partial removal of the meniscus. Now, clinicians are shifting toward preserving the biomechanical function of the medial meniscus through root repair. While good outcomes have been reported for this surgical treatment option, relatively few studies have compared root repair with other treatment methods. Authors of a recent study compared the long-term outcomes of nonoperative management or partial meniscectomy with those of root repair by the transtibial pull-through technique. For a matched cohort of 45 patients, the team looked at rates of arthritis progression, subsequent revision knee arthroplasty, International Knee Documentation and Tegner scores, and radiographic outcomes. With a mean follow-up of 74 months, they observed significant differences in the rate of arthritis progression and subsequent knee arthroplasty among the treatment groups. Notably, no patients in the meniscal repair group demonstrated a need for arthroplasty. Additionally, the repair group showed significantly less arthritic progression compared to the nonoperative and partial meniscectomy group, as measured by changes in Kellgren-Lawrence grade from pre-op to post-op. The findings suggest that for middle-aged patients with a medial meniscus posterior root tear, repair is a favorable treatment option for preventing further arthritis and the need for knee replacement surgery. However, more studies are needed to help clinicians further define the ideal individualized treatment option for patients with meniscal root tears based on their clinical, demographic and radiographic characteristics. 